\title{
RECEIVED
}

Allf 121997

OSTI

\section{Single Identities for Lattice Theory and for Weakly Associative Lattices}

\author{
W. McCune* \\ Wathematics and Computer Science Division \\ Argonne National Laboratory \\ Argonne, Illinois 60439-4844 \\ U.S.A. \\ R. Padmanabhan ${ }^{\dagger}$ \\ Department of Mathematics \\ University of Manitoba \\ Winnipeg, Manitoba R3T 2N2 \\ Canada
}

March 13, 1995

\begin{abstract}
We present a single identity for the variety of all lattices that is much simpler than those previously known to us. We also show that the variety of weakly associative lattices is one-based, and we present a generalized one-based theorem for subvarieties of weakly associative lattices that can be defined with absorption laws. The automated theorem-proving program OTTER was used in a substantial way to obtain the results.
\end{abstract}

\section{Introduction}

Equational identities are, perhaps, the simplest form of sentences expressing many basic properties of algebras. Several familiar classes of algebras, such as semigroups, groups, rings, lattices, and Boolean algebras, are defined by equational identities. Such a class of algebras is known as an equational

\footnotetext{
-Supported by the Office of Scientific Computing, U.S. Department of Energy, under Contract W-31-109-Eng-38.

'Supported by an operating grant from NSERC of Canada (\#A8215).
} 
class of algebras or a variety of algebras (for mathematical properties of such classes, see [3], [10], and [11]).

An equational theory $T$ is said to be finitely based if it can be defined by a finite set of identities. If an equational theory $T$ is finitely based, we may wish to determine the least number of equations needed to define the theory $T$, in particular, to determine whether $T$ is one-based. It is known that every finitely based variety of lattices is two-based and that it is onebased iff it is either the variety of all lattices or else the trivial variety of one-element lattices defined by $x=y$ (cf. [7] and [8]).

This paper has two parts. First, we improve several results on the variety of all lattices. In particular, we simplify McKenzie's absorption basis for lattice theory, present a reduction schema containing two unary functions for single identities, and find a single identity for lattice theory much shorter than those previously reported. Second, we show that the theory of weakly associative lattices is one-based and that every subvariety of weakly associative lattices that is definable with absorption laws is one-based.

The automated theorem-proving program OTTER [6] was used in a substantial way to obtain the results in this paper, and we present some of the proofs found by OTTER. Because the practical use of automated theorem proving is new and not widely known, we also indicate how OTTER was used. In many cases, our goal was to find identities that have particular properties as well as to prove properties of identities; in some of those cases, we used OTTER to generate a large set of candidates and ran OTTER searches with members or subsets of the candidates. We stress that OTTER was not used as a proof checker; rather, it searched for proofs, and we think of those proofs as OTTER's.

\section{Single Identities for Lattice Theory}

An absorption equality has a variable as one side of the equality symbol and a term with at least one other variable as the other side. Most "nice" varieties satisfy absorption laws, for example,

$$
\begin{aligned}
& x-(x-y)=y, \quad \text { in Abelian groups and rings, } \\
& x \wedge(x \vee y)=x, \quad \text { in lattices. }
\end{aligned}
$$

In contrast, the variety of all semigroups does not satisfy any absorption law. 
If a lattice variety is one based, it must be definable by a single absorption law, and as a prelude, we must have a basis consisting entirely of absorption identities. The simplest previously known absorption basis for lattice theory (in terms of $\wedge$ and $\vee$ ) was McKenzie's [7], consisting of the four identities

$$
\begin{gathered}
y \vee(x \wedge(y \wedge z))=y, \\
y \wedge(x \vee(y \vee z))=y, \\
((x \wedge y) \vee(y \wedge z)) \vee y=y, \\
((x \vee y) \wedge(y \vee z)) \wedge y=y .
\end{gathered}
$$

McKenzie first constructed a single identity, containing 34 variables, for lattice theory [7]; then G. Grätzer asked for a short identity defining lattices [2]. Padmanabhan's reduction schema [9], along with McKenzie's absorption basis above, yields a single identity with just seven variables; however, written without abbreviation, it has length 355 (where length is the count of symbols, including variables, " $\wedge ", " \vee "$, and $"="$, but not parentheses).

\subsection{A Simpler Absorption Basis for Lattice Theory}

The program MACE [5], which searches for models and counterexamples, was used to show that McKenzie's absorption basis (1)-(4) is independent. The automated theorem-proving program OTTER [6] was then used to examine variants of $(1)-(4)$ in which terms are commuted. After many OTTER searches, it was discovered that the pair (1) and (4) can be replaced with the following variant of (4):

$$
((y \vee x) \wedge(y \vee z)) \wedge y=y .
$$

Lemma 1 The set of identities $\{(2),(3),(5)\}$ is a basis for the variety of all lattices.

We list here an equational proof found by OTTER, starting with $\{(2)$, $(3),(5)\}$ and deriving $\{(1),(4)\}$. The justification " $m \rightarrow n$ " indicates paramodulation from $m$ into $n$, that is, equality substitution, using (an instance of) $m$, into a subterm of (an instance of) $n ; ": i, j, \ldots$ " indicates simplification with $i, j, \ldots ;$ and "flip" indicates that the equation is reversed so that the more complex side is on the left.

Proof (found by OTTER 3.0.3d on gyro at 3.33 seconds). 


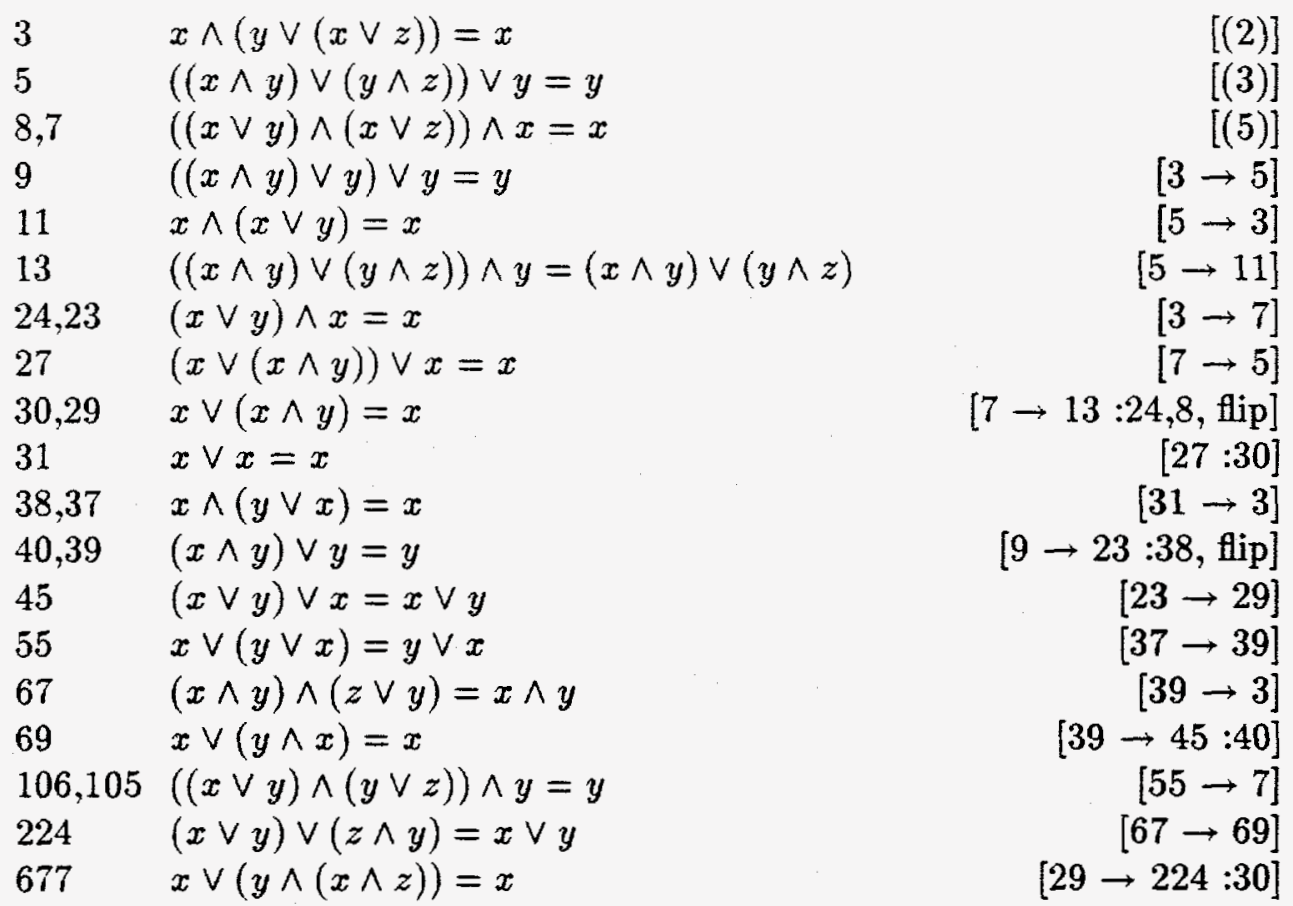

Line 106 is (4), and line 677 is (1).

\subsection{A New Reduction Schema}

Padmanabhan's reduction schema in [9] is

$$
p(p(x, y, y), u, p(p(x, y, y), f(y), z))=y,
$$

where $p$ is a majority polynomial. This is equivalent to the set

$$
\{p(y, y, x)=y, p(y, x, y)=y, p(x, y, y)=y, f(y)=y\} .
$$

(To build a single identity for a theory satisfying a majority polynomial and axiomatized with an absorption basis, say of size $n$, one can apply the lemma in [9] $n-1$ times, then substitute the result in the reduction schema.)

Lemma 2 The identity

$$
p(p(x, y, y), p(x, p(y, z, f(y)), g(y)), u)=y
$$


is equivalent to the set

$$
\{p(y, y, x)=y, p(y, x, y)=y, p(x, y, y)=y, f(y)=y, g(y)=y\} .
$$

Proof (found by OTTER 3.0.3d on gyro at 0.28 seconds).

$$
\begin{array}{llr}
4,3 & p(p(x, y, y), p(x, p(y, z, f(y)), g(y)), u)=y & {[(8)]} \\
5 & p(p(x, p(y, z, z), p(y, z, z)), p(x, z, g(p(y, z, z))), u)=p(y, z, z)[3 \rightarrow 3] \\
7 & p(p(x, y, y), p(x, p(z, p(y, u, f(y)), g(y)), g(y)), v)=y[3 \rightarrow 5: 4,4,4] \\
10,9 & p(p(p(x, y, y), y, y), y, z)=y & {[3 \rightarrow 7]} \\
16,15 & p(x, x, y)=x & {[9 \rightarrow 9]} \\
19,18 & p(p(x, y, y), p(x, y, g(y)), z)=y & {[9 \rightarrow 5: 10,10,10]} \\
22 & p(p(x, y, y), p(x, p(z, y, g(y)), g(y)), u)=y & {[15 \rightarrow 7]} \\
33,32 & p(x, y, g(y))=y & {[18 \rightarrow 18: 19,16, \text { flip] }} \\
40 & p(x, p(y, z, f(y)), g(y))=y & {[3 \rightarrow 18: 4,16, \text { flip] }} \\
42 & p(p(x, y, y), y, z)=y & {[22: 33,33]} \\
44 & p(x, y, f(x))=x & {[42 \rightarrow 40]} \\
47,46 & p(x, y, y)=y & {[42 \rightarrow 44, \text { flip] }} \\
50,49 & f(x)=x & {[44 \rightarrow 46, \text { flip] }} \\
53,52 & p(x, y, x)=x & {[44: 50]} \\
55 & g(x)=x & {[32 \rightarrow 52, \text { flip] }}
\end{array}
$$

Lines $16,47,50,53$, and 55 establish one direction of the equivalence; the other is established by inspection.

The reduction schema (8) was found by automatically generating candidate schemas with OTTER, then with each, searching with OTTER for a proof of the preceding type.

With the new reduction schema, because we have $f(y)=y$ and $g(y)=y$, we apply the lemma of [9] $n-2$ times instead of $n-1$ times. With the new lattice theory absorption basis of size $n=3$, instead of $n=4$, we can build a single identity with just one application of the lemma. Using a variant of the majority polynomial used to build the length 355 identity, we can build a single identity of length 139 , again with 7 variables, for lattice theory.

\subsection{A Simpler Single Identity for Lattice Theory}

The reduction schema (8) and the majority polynomial

$$
p(x, y, z)=(x \wedge z) \vee(y \wedge(x \vee z))
$$


satisfy the three identities

$$
\begin{aligned}
& (x \wedge y) \vee(x \wedge(x \vee y))=x, \\
& (x \wedge x) \vee(y \wedge(x \vee x))=x, \\
& (x \wedge y) \vee(y \wedge(x \vee y))=y .
\end{aligned}
$$

To simplify construction of a single identity (producing a shorter one), we can use the fact that these identities are built in to the reduction schema. In particular, if an absorption basis for lattice theory contains the above three identities, those three identities need not be used in the construction of a single identity.

Lemma 3 The absorption identities

$$
\begin{gathered}
((x \wedge y) \vee(y \wedge z)) \vee y=y \\
((x \vee(y \vee z)) \wedge(u \vee y)) \wedge y=y
\end{gathered}
$$

union the three built-in identities $\{(11),(12),(13)\}$ are a basis for the variety of all lattices.

Note that (14) is the same as (3).

Proof (found by OTTER $3.0 .3 \mathrm{~d}$ on gyro at 0.82 seconds).

5

7

9

11

14,13

15

19

26,25

27

31

39

41

47

51

$$
\begin{aligned}
& (x \wedge y) \vee(x \wedge(x \vee y))=x \\
& (x \wedge x) \vee(y \wedge(x \vee x))=x \\
& (x \wedge y) \vee(y \wedge(x \vee y))=y \\
& ((x \wedge y) \vee(y \wedge z)) \vee y=y
\end{aligned}
$$$$
((x \vee(y \vee z)) \wedge(u \vee y)) \wedge y=y
$$$$
x \vee x=x
$$$$
(x \wedge x) \vee(y \wedge x)=x
$$$$
((x \wedge y) \vee(y \wedge z)) \wedge y=(x \wedge y) \vee(y \wedge z)
$$$$
x \wedge x=x
$$$$
x \vee(y \wedge x)=x
$$$$
(x \vee(x \wedge y)) \vee x=x
$$$$
((x \vee y) \wedge(z \vee x)) \wedge x=x
$$$$
((x \vee(y \vee z)) \wedge y) \wedge y=y
$$$$
(x \wedge y) \vee y=y
$$$$
(x \wedge(y \wedge x)) \vee x=x
$$

$[15: 26]$

$[25 \rightarrow 9]$

$[13 \rightarrow 11]$

$[13 \rightarrow 11]$

$[27 \rightarrow 9]$

$[27 \rightarrow 3: 26]$ 


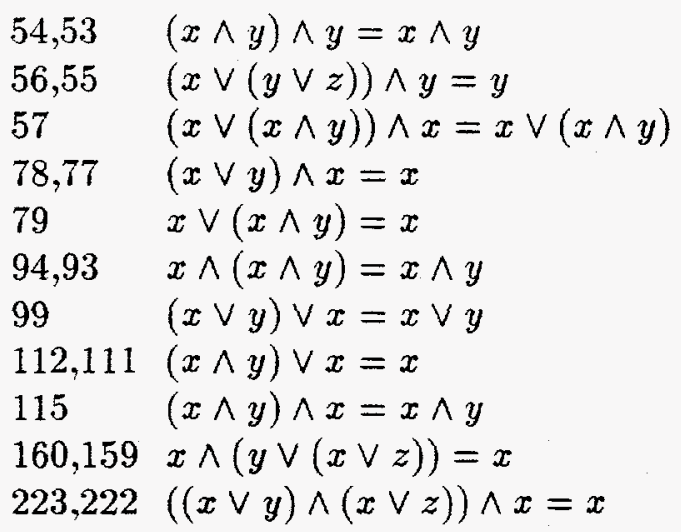

$[47 \rightarrow 3: 14]$

$[31 \rightarrow 3: 14]$

$[51 \rightarrow 55]$

$[57: 78$, flip]

$[3 \rightarrow 77]$

$[77 \rightarrow 79]$

$[79 \rightarrow 3: 94,26]$

$[111 \rightarrow 19: 112]$

$[55 \rightarrow 115: 56]$

$[99 \rightarrow 39]$

Lines 9,160 , and 223 are (3), (2), and (5), respectively.

The pair (14) and (15) was found by automatically searching with many candidate sets for proofs of the preceding type.

Theorem 1 The identity

$$
\begin{aligned}
& (((x \wedge y) \vee(y \wedge(x \vee y))) \wedge z) \vee\left(\left(\left(x \wedge \left(\left(\left(x_{1} \wedge y\right) \vee\left(y \wedge x_{2}\right)\right) \vee\right.\right.\right.\right. \\
& y)) \vee\left(\left(\left(y \wedge\left(\left(\left(x_{1} \vee\left(y \vee x_{2}\right)\right) \wedge\left(x_{3} \vee y\right)\right) \wedge y\right)\right) \vee(u \wedge(y \vee\right.\right. \\
& \left.\left.\left(\left(\left(x_{1} \vee\left(y \vee x_{2}\right)\right) \wedge\left(x_{3} \vee y\right)\right) \wedge y\right)\right)\right) \wedge\left(x \vee \left(\left(\left(x_{1} \wedge y\right) \vee(y \wedge\right.\right.\right. \\
& \left.\left.\left.\left.\left.\left.\left.x_{2}\right)\right) \vee y\right)\right)\right)\right) \wedge(((x \wedge y) \vee(y \wedge(x \vee y))) \vee z)\right)=y
\end{aligned}
$$

is a single identity for the variety of all lattices.

Proof. With (14) and (15), we need not apply the lemma in [9] to build a single identity. With the reduction schema (8) we can simply substitute (14) and (15) for $f(y)$ and $g(y)$. The result, written in terms of $\wedge$ and $\vee$, is (16). (It has length 79 , again with 7 variables.)

\section{$3 \quad$ Weakly Associative Lattices}

Here we prove that the variety of all weakly associative lattices (WAL) is one-based. We then show that every finitely based subvariety of WAL that is definable by absorption laws is also one-based. 


\subsection{WAL is One-based}

We take as our starting point the following basis for WAL [1].

$$
\begin{array}{lll}
x \wedge x=x, & x \vee x=x, & \left(\mathrm{~W} 1, \mathrm{~W} 1^{\prime}\right) \\
x \wedge y=y \wedge x, & x \vee y=y \vee x, & \left(\mathrm{~W} 2, \mathrm{~W} 2^{\prime}\right) \\
((x \vee z) \wedge(y \vee z)) \wedge z=z, & ((x \wedge z) \vee(y \wedge z)) \vee z=z, & \left(\mathrm{~W} 3, \mathrm{~W} 3^{\prime}\right) \\
(x \vee z) \wedge z=z, & (x \wedge z) \vee z=z . & \left(\mathrm{W} 4, \mathrm{~W} 4^{\prime}\right)
\end{array}
$$

Clearly, (W4) follows from (W1) and (W3), and dually, (W4') follows from $\left(\mathrm{W} 1^{\prime}\right)$ and $\left(\mathrm{W} 3^{\prime}\right)$; henceforth we omit (W4,W4').

The ternary polynomial (10) is a majority polynomial for WAL as well as for lattice theory. Therefore, if we can find an absorption basis for WAL, we can construct a single identity for WAL. Since the built in identities (11)-(13) hold for WAL, we can attempt the same sort of construction as for lattice theory in Sec. 2.3.

With a sequence of OTTER searches, we tested many pairs of absorption identities, adding each pair to (11)-(13) and attempting to derive the set (W1, W1', W2, W2', W3, W3'). OTTER succeeded with the pair

$$
\begin{aligned}
& ((x \vee y) \wedge(z \vee x)) \wedge x=x, \\
& ((x \wedge y) \vee(z \wedge x)) \vee x=x
\end{aligned}
$$

Lemma 4 The set $\{(11),(12),(13),(17),(18)\}$ is a basis for the variety of all weakly associative lattices.

Because (17) and (18) are simply commuted variants of (W3) and (W3'), it is sufficient to derive idempotence and commutativity of the two operations.

Proof (found by OTTER $3.0 .3 \mathrm{~d}$ on gyro at 2.51 seconds).

4

6

8

10

12

14

$$
\begin{aligned}
& (x \wedge y) \vee(x \wedge(x \vee y))=x \\
& (x \wedge x) \vee(y \wedge(x \vee x))=x \\
& (x \wedge y) \vee(y \wedge(x \vee y))=y \\
& ((x \vee y) \wedge(z \vee x)) \wedge x=x \\
& ((x \wedge y) \vee(z \wedge x)) \vee x=x \\
& (x \wedge(y \vee(z \wedge x))) \wedge(z \wedge x)=z \wedge x
\end{aligned}
$$

$[8 \rightarrow 10]$ 


\begin{tabular}{|c|c|c|}
\hline 16 & $(x \wedge(y \vee(x \wedge z))) \wedge(x \wedge z)=x \wedge z$ & {$[4 \rightarrow 10]$} \\
\hline 21,20 & $x \vee(x \wedge(((x \vee y) \wedge(z \vee x)) \vee x))=x$ & {$[10 \rightarrow 8]$} \\
\hline 24 & $((x \wedge y) \vee x) \vee x=x$ & {$[10 \rightarrow 12]$} \\
\hline 27,26 & $((x \vee y) \wedge x) \wedge x=x$ & {$[12 \rightarrow 10]$} \\
\hline 30 & $(((x \wedge y) \vee x) \wedge x) \vee(x \wedge x)=x$ & {$[24 \rightarrow 8]$} \\
\hline 34 & $(x \wedge(y \wedge x)) \wedge(y \wedge x)=y \wedge x$ & {$[8 \rightarrow 26]$} \\
\hline 45,44 & $(x \wedge x) \wedge x=x$ & {$[26 \rightarrow 34: 27,27]$} \\
\hline 47,46 & $(x \wedge(y \vee x)) \wedge x=x$ & {$[26 \rightarrow 14: 27,27]$} \\
\hline 48 & $(x \wedge x) \wedge(x \wedge x)=x \wedge x$ & {$[30 \rightarrow 14]$} \\
\hline 63,62 & $x \wedge(x \wedge(y \vee x))=x \wedge(y \vee x)$ & {$[8 \rightarrow 46: 47]$} \\
\hline 64 & $x \vee(x \wedge((x \wedge(y \vee x)) \vee x))=x$ & {$[46 \rightarrow 8]$} \\
\hline 70 & $((x \wedge x) \vee(x \wedge x)) \vee(x \wedge x)=x \wedge x$ & {$[48 \rightarrow 24]$} \\
\hline 74 & $((x \wedge x) \wedge(y \vee x)) \wedge x=x$ & {$[44 \rightarrow 16: 45,45]$} \\
\hline 89,88 & $x \wedge x=x$ & {$[24 \rightarrow 74: 45]$} \\
\hline 95,94 & $(x \vee x) \vee x=x$ & {$[70: 89,89,89,89]$} \\
\hline 106 & $x \vee(y \wedge(x \vee x))=x$ & {$[6: 89]$} \\
\hline 114,113 & $x \vee x=x$ & {$[88 \rightarrow 20: 95,89]$} \\
\hline 116 & $x \vee(y \wedge x)=x$ & {$[106: 114]$} \\
\hline 119,118 & $(x \wedge(((x \vee y) \wedge(z \vee x)) \vee x)) \vee x=x$ & {$[20 \rightarrow 4: 63,89]$} \\
\hline 123,122 & $x \wedge(y \wedge x)=y \wedge x$ & {$[116 \rightarrow 14: 89]$} \\
\hline 128 & $(x \wedge y) \vee y=y$ & {$[116 \rightarrow 4: 123,89]$} \\
\hline 131,130 & $(x \wedge y) \wedge y=x \wedge y$ & {$[128 \rightarrow 4: 114]$} \\
\hline 134 & $(x \vee y) \wedge x=x$ & {$[26: 131]$} \\
\hline 136 & $x \wedge(((x \vee y) \wedge(z \vee x)) \vee x)=x$ & {$[20 \rightarrow 64: 47,21,47,119$, flip $]$} \\
\hline 141,140 & $x \wedge((x \wedge y) \vee x)=(x \wedge y) \vee x$ & {$[24 \rightarrow 134]$} \\
\hline 142 & $x \vee((x \wedge(y \vee x)) \vee x)=x$ & {$[64: 141]$} \\
\hline 144 & $(x \vee(x \vee y)) \vee(x \vee y)=x \vee y$ & {$[134 \rightarrow 24]$} \\
\hline 152 & $((x \wedge y) \vee(z \wedge y)) \vee y=y$ & {$[122 \rightarrow 12]$} \\
\hline 172 & $(x \wedge(y \vee x)) \vee x=x$ & {$[142 \rightarrow 136: 141]$} \\
\hline 179,178 & $x \wedge(y \vee x)=x$ & {$[172 \rightarrow 4: 47,47,114$, flip $]$} \\
\hline 180 & $(x \wedge y) \vee x=x$ & {$[140: 179$, flip $]$} \\
\hline 189,188 & $(x \vee y) \vee y=x \vee y$ & {$[178 \rightarrow 116]$} \\
\hline 194 & $(x \vee(y \wedge(z \vee x))) \vee(z \vee x)=z \vee x$ & {$[178 \rightarrow 152]$} \\
\hline 198 & $x \vee(x \vee y)=x \vee y$ & {$[144: 189]$} \\
\hline 200 & $(x \wedge y) \wedge x=x \wedge y$ & {$[180 \rightarrow 4: 114]$} \\
\hline 212 & $x \wedge(x \vee y)=x$ & {$[198 \rightarrow 4: 114]$} \\
\hline 216 & $(x \vee y) \vee x=x \vee y$ & {$[212 \rightarrow 116]$} \\
\hline 236 & $x \vee(x \wedge y)=x$ & {$[200 \rightarrow 116]$} \\
\hline
\end{tabular}


$243,242(x \wedge y) \wedge(y \wedge x)=y \wedge x$

$[236 \rightarrow 14]$

$286 x \wedge y=y \wedge x$

$288,287(x \vee y) \vee(y \vee x)=y \vee x$

$[242 \rightarrow 200: 243,243]$

$[212 \rightarrow 194]$

$349 \quad x \vee y=y \vee x$

$[287 \rightarrow 216: 288,288]$

Lines $89,114,286$, and 349 establish the result.

Theorem 2 The variety all weakly associative lattices is one-based.

Proof. Using the reduction schema (8), we can construct a single identity for WAL by substituting (17) and (18) for $f(y)$ and $g(y)$. The identity, written in terms of $\wedge$ and $\vee$, has length 75 and 6 variables:

$$
\begin{aligned}
& (((x \wedge y) \vee(y \wedge(x \vee y))) \wedge z) \vee\left(\left(\left(x \wedge \left(\left(\left(y \wedge x_{1}\right) \vee\left(x_{2} \wedge y\right)\right) \vee\right.\right.\right.\right. \\
& y)) \vee\left(\left(( y \wedge ( ( ( y \vee x _ { 1 } ) \wedge ( x _ { 2 } \vee y ) ) \wedge y ) ) \vee \left(u \wedge \left(y \vee \left(\left(\left(y \vee x_{1}\right) \wedge\right.\right.\right.\right.\right.\right. \\
& \left.\left.\left.\left.\left.\left.\left.\left(x_{2} \vee y\right)\right) \wedge y\right)\right)\right)\right) \wedge\left(x \vee\left(\left(\left(y \wedge x_{1}\right) \vee\left(x_{2} \wedge y\right)\right) \vee y\right)\right)\right)\right) \wedge(((x \wedge \\
& y) \vee(y \wedge(x \vee y))) \vee z))=y
\end{aligned}
$$

\subsection{Subvarieties of WAL}

Let gWAL stand for the equational theory of all WALs satisfying a further absorption law, say $g\left(x_{1}, x_{2}, \cdots, x_{n}\right)=x_{1}$. To find a single identity for gWAL, we must embed the additional absorption law into a single identity for WAL. The idea of embedding a unary function inside an identity goes back to G. Higman and B. H. Neumann (see [4] where they prove that every finitely based variety of group theory is one-based). Such a technique was first applied to lattices by Padmanabhan in [8].

Theorem 3 The variety gWAL is one-based.

Proof. We use the reduction schema (8), which has two unary functions,

$$
p(p(x, y, y), p(x, p(y, z, f(y)), g(y)), u)=y,
$$

and we write (17) and (18) as $W_{1}(y)=y$ and $W_{2}(y)=y$. Using the lemma in [9], we bundle the identities (17) and (18) as $p\left(W_{1}(y), W_{2}(y), x\right)$, and substitute this for $f(y)$; we keep $g(y)$ as a place holder for the further 
absorption law $g\left(x_{1}, x_{2}, \cdots, x_{n}\right)=x_{1}$. The resulting single identity, written in terms of $\Lambda$ and $V$ (using the same majority polynomial as above) is

$$
\begin{aligned}
& (((x \wedge y) \vee(y \wedge(x \vee y))) \wedge z) \vee\left(\left(( x \wedge g ( y ) ) \vee \left(\left(\left(y \wedge \left(\left(\left(\left(\left(y \vee x_{1}\right) \wedge\right.\right.\right.\right.\right.\right.\right.\right.\right. \\
& \left.\left.\left.\left(x_{2} \vee y\right)\right) \wedge y\right) \wedge u\right) \vee\left(\left(\left(\left(y \wedge x_{1}\right) \vee\left(x_{2} \wedge y\right)\right) \vee y\right) \wedge((((y \vee\right. \\
& \left.\left.\left.\left.\left.\left.\left.x_{1}\right) \wedge\left(x_{2} \vee y\right)\right) \wedge y\right) \vee u\right)\right)\right)\right) \vee\left(v \wedge \left(y \vee \left(\left(\left(\left(( y \vee x _ { 1 } ) \wedge \left(x_{2} \vee\right.\right.\right.\right.\right.\right.\right. \\
& y)) \wedge y) \wedge u) \vee\left(( ( ( y \wedge x _ { 1 } ) \vee ( x _ { 2 } \wedge y ) ) \vee y ) \wedge \left(\left(\left(\left(y \vee x_{1}\right) \wedge\right.\right.\right.\right. \\
& \left.\left.\left.\left.\left.\left.\left.\left.\left.\left(x_{2} \vee y\right)\right) \wedge y\right) \vee u\right)\right)\right)\right)\right) \wedge(x \vee g(y))\right)\right) \wedge(((x \wedge y) \vee(y \wedge(x \vee \\
& y))) \vee z))=y .
\end{aligned}
$$

The preceding single identity for gWAL has length 109 and 7 variables. Note the two occurrences of $g(y)$, which represent the further absorption law. If a further set of absorption laws, instead of a single law, is added, the set can be bundled, as above, with applications of the lemma in [9].

The theory of all lattices may be viewed as a weakly associative lattice satisfying the additional absorption law

$$
x \wedge(y \vee(x \vee z))=x .
$$

Lemma $5\{\mathrm{WAL},(21)\} \Rightarrow\{$ the two associative laws for $\wedge$ and $\vee\}$.

Proof. Define $x \leq y$ to mean $x \wedge y=x$. By the commutative law, $x \leq y$ and $y \leq x$ imply that $x=y$. Also, if $x \leq y$, then $x \vee y=(x \wedge y) \vee y=y$. Let $x \leq y$ and $y \leq z$. Then

$$
x \wedge z=x \wedge(z \vee y)=x \wedge(z \vee(x \vee y))=x
$$

and hence $x \leq z$. Therefore, the binary relation " $\leq$ " is reflexive, antisymmetric, and transitive. Now, by the commutative and absorption laws, $x \wedge y$ is a lower bound of $x$ and $y$. Let $u$ be any lower bound of $x$ and $y$, that is, let $u \leq x, u \leq y$. In other words, $x=u \vee x$ and $y=u \vee y$. We have

$$
u \wedge(x \wedge y)=u \wedge((u \vee x) \wedge(u \vee y))=u
$$

by (W3), and hence $u \leq x \wedge y$. In other words, $x \wedge y$ is the greatest lower bound of the set $\{x, y\}$. Thus the meet operation " $\wedge$ " is associative. This is where the mathematician becomes informal and jumps to the conclusion

$$
(x \wedge y) \wedge z=\text { the g.l.b. of }\{\{x, y\}, z\}=\text { g.l.b. of }\{x, y, z\}=x \wedge(y \wedge z) .
$$

The dual argument (after transitivity of " $\leq$ ") holds for associativity of " $V$ ". 
(Lemma 5 was also proved by OTTER, but that proof is too complex to present here.)

Therefore, as a corollary to Theorem 3 , we have that lattice theory is one-based (cf. [7], [9], and Sec. 2).

\section{Open Questions}

1. Are there single identities for lattice theory, WAL, or gWAL simpler than the ones presented here $(16,19,20)$ ?

2. Is the variety of near lattices (NL) one-based? A basis for NL is the following set:

$$
\begin{array}{ll}
x \wedge x=x, & x \vee x=x, \\
x \wedge y=y \wedge x, & x \vee y=y \vee x, \\
x \wedge(x \vee y)=x, & x \vee(x \wedge y)=x
\end{array}
$$

3. Is the variety of transitive near lattices (TNL) one-based? We have a basis for TNL by adjoining the following pair to NL:

$$
x \wedge(y \vee(x \vee z))=x, \quad x \vee(y \wedge(x \wedge z))=x \text {. }
$$

\section{References}

[1] E. Fried and G. Grätzer. Some examples of weakly associative lattices. Colloq. Math., 27:215-221, 1973.

[2] G. Grätzer. Lattice theory: First concepts and distributive lattices. San Francisco, 1971.

[3] G. Grätzer. Universal Algebra. Springer Verlag, 2nd edition, 1979.

[4] G. Higman and B. H. Neumann. Groups as groupoids with one law. Publicationes Mathematicae Debrecen, 2:215-227, 1952.

[5] W. McCune. A Davis-Putnam program and its application to finite first-order model search: Quasigroup existence problems. Tech. Report ANL/MCS-TM-194, Argonne National Laboratory, Argonne, Ill., May 1994. 
[6] W. McCune. OTter 3.0 reference manual and guide. Tech. Report ANL-94/6, Argonne National Laboratory, Argonne, Ill., 1994.

[7] R. N. McKenzie. Equational bases for lattice theories. Math. Scand., $27: 24-38,1970$.

[8] R. Padmanabhan. Two identities for lattices. Proc. Amer. Math. Soc., 20:409-412, 1969.

[9] R. Padmanabhan. Equational theory of algebras with a majority polynomial. Algebra Universalis, 7(2):273-275, 1977.

[10] A. Tarski. Equational logic and equational theories of algebras. In K. Schütte, editor, Contributions to Mathematical Logic, pages 275288. North-Holland, Amsterdam, 1968.

[11] W. Taylor. Equational logic. Appendix 4 in G. Grätzer, Universal Algebra. Springer-Verlag, 1979.

\section{DISCLAIMER}

This report was prepared as an account of work sponsored by an agency of the United States Government. Neither the United States Government nor any agency thereof, nor any of their employees, makes any warranty, express or implied, or assumes any legal liability or responsibility for the accuracy, completeness, or usefulness of any information, apparatus, product, or process disclosed, or represents that its use would not infringe privately owned rights. Reference herein to any specific commercial product, process, or service by trade name, trademark, manufacturer, or otherwise does not necessarily constitute or imply its endorsement, recom. mendation, or favoring by the United States Government or any agency thereof. The views and opinions of authors expressed herein do not necessarily state or reflect those of the United States Government or any agency thereof. 


\section{Appendix A}

The programs OTTER and MACE are in the public domain and are available through Internet by FTP. See the file

ftp://info.mcs.anl.gov/pub/Otter/README

for more information.

Several OTTER input files and the corresponding proofs are available by FTP. These include input files for the OTTER proofs that appear here, input files for the OTTER proof of Lemma 5, and input files that were used to double check, directly, that (16), (19), and (20) are single identities for lattice theory, WAL, and gWAL, respectively. See

ftp://info.mcs.anl.gov/pub/Otter/LT-WAL/README

for specific information on the input files. 


\section{Appendix B}

Figures 1,2 , and 3 are the single identities 16,19 , and 20 , respectively, displayed in two dimensions, with meet operations vertical, join operations horizontal, and boxes instead of parentheses. The purpose of these figures is simply to show the structure of the identities.

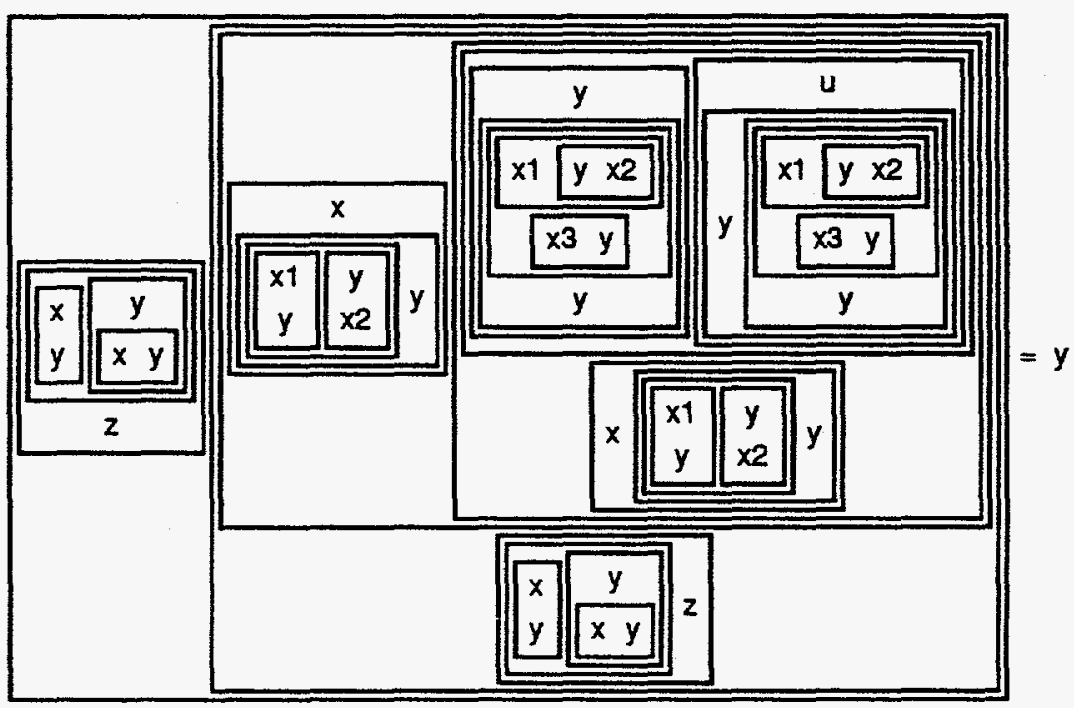

Figure 1: LT Single Identity (16) 


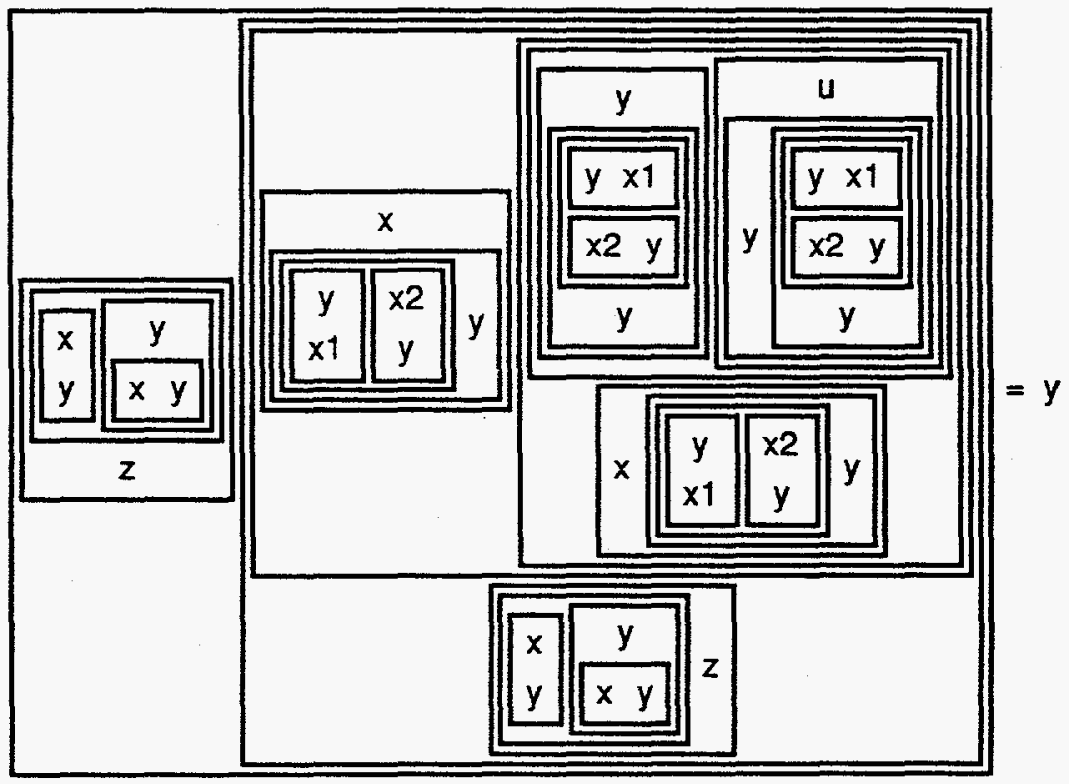

Figure 2: WAL Single Identity (19)

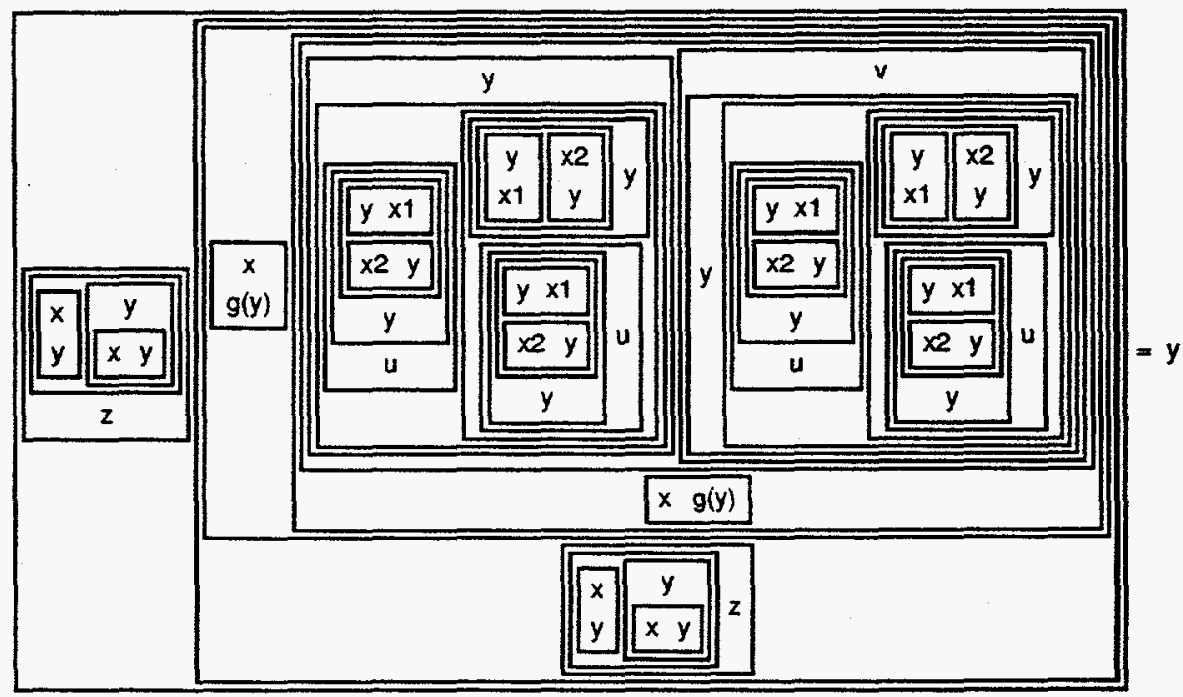

Figure 3: gWAL Single Identity (20) 


\section{DISCLAMIER}

Portions of this document may be illegible in electronic image products. Images are produced from the best available original document. 